\title{
PERFORMANCE OF NETWORK CODING SCHEMES FOR 5G SYSTEM
}

\author{
Shurooq M. Abdulkhudhur ${ }^{1}$, Abdulkareem A. Kadhim ${ }^{2}$ \\ ${ }^{1,2}$ College of Information Engineering, Al-Nahrain University, Baghdad, Iraq \\ \{ shorooq.alrubaye, abdulkareem.a\}@ coie-nahrain.edu.iq ${ }^{1,2}$ \\ Received:18/2/2021, Accepted:19/3/2021
}

\begin{abstract}
Huge data rates have been provided by $5 \mathrm{G}$ wireless communication systems using a millimetre wave (mmWave) band that have frequencies ranging from 30 to $300 \mathrm{GHz}$. mmWave provides about 10 times the bandwidth used in the existing 4G system. The 5G network deals with a massive number of devices. This presents many challenges including capacity, data rate, end to end delay, and a very large number of connections. In this paper, the main task is to apply network coding to a $5 \mathrm{G}$ mmave communication system to increase the throughput of the communication links. Simple packet-based network coding schemes using butterfly network topology are simulated. The two network coding schemes considered here are Physical Layer Network Coding (PLNC) and Network Layer Network Coding (NLNC). Models of Additive White Gaussian Noise (AWGN) and mmWave indoor fading channels are considered in the work using Quadrature Phase Shift Keying (QPSK) modulation. The results of the tests show that the use of both NLNC and PLNC improved throughput in comparison to the uncoded system. Using PLNC increased the Bit Error Rate (BER) and the Packet Error Rate (PER), while the NLNC scheme shows almost identical error performance to the uncoded system over the mmWave fading channel. The NLNC presents better throughput performance, measured in Bits/s, by about $14 \%$ over the uncoded system, whereas in terms of Packets/s, both the network coded and uncoded systems have approximately identical throughput performance at very low SNR for the mmWave channel. At high SNR, the results show that network coding improved throughput when compared to the non-coding case by about $25 \%$ at the expense of a slightly increased error rate.
\end{abstract}

keywords: Network coding, Millimeter-wave, Throughput, 5G system.

\section{INTRODUCTION}

Compared to the 4G system, 5G networks are characterized by; 1000 times the system capacity, wide frequency band covering microwave and millimeter wave (mmWave) frequency bands, up to 100 times data rate, reduced latency, and better energy consumption [1]-[5]. Network coding (NC) is an active research topic in information theory that affects the traditional concept of routing. Rather than simply transmitting data or packets, intermediate nodes may be used to recombine multiple input packets into one or more output packets to achieve optimal information flow [6]. Network coding approaches were shown to enhance network throughput, improve network robustness, reduce consumed energy, and achieve the network capacity [7], [8]. The author in [9] studied the approach of combined network coding with MIMO technology which was examined with different wireless channel models. The first implementation of a two-way relay network based on physical-layer network coding (PLNC) is presented in [10]. Reed Solomon (RS) code was used with network coding for Wireless Sensor Networks (WSNs) over fading channels using butterfly network topology [11]. The characteristic, benefits, scenarios, and applications of network coding for $5 \mathrm{G}$ network and device to device communication are presented and evaluated in [12]. Network coding schemes integrated with 5G new Radio Access Networks (RAN) that improve reliability and radio efficiency of industrial applications [13]. PLNC that effectively enhance the throughput of wireless networks by mapping superimposed signals at the receiver to other forms of user messages is presented in [14]. Theoretical study of massive Multi-Input Multi-Output (MIMO) with physical-layer network coding using a different number of users and MIMO dimensionality in the multiuser environment were presented in [15]. In general, the previous related works relied 
on using of PLNC only for 5G to improve the system robustness and error performance. In this work, a comparative study of PLNC and NLNC when used in 5G environment is presented. The main intention here is to increase the system throughput via the use of different network coding schemes. This work aims is to improve the throughput of the system by using network coding in the mmWave band using two approaches: PLNC and NLNC. These are considered for the case of backhaul indoor $5 \mathrm{G}$ environment, where the network coding will be applied at the relay node to reduce the number of transmissions and improve the reliability of mmWaves communication links. The remaining parts of the paper are organized as follows; in Section-II, the model of the system is presented with the topology of the network together with the main assumptions and transmission parameters used in the work. The third section presents the simulation tests and their results in the form of bit and packet error rated and throughput performance versus signal to noise power ratio (SNR). Finally, Section-IV deals with the main concluding remarks of the work.

\section{SYSTEM MODEL}

The main task of this work is to use network coding over a butterfly network topology. This network represents a typical example of a topology that consists of a source, destination, and relay nodes. It is usually considered in network coding works where its model allows cooperative communication between all nodes. The considered butterfly topology consists of five nodes: two source nodes (S1 and S2), two destination nodes (D1 and D2), and one relay node (R). Each source and relay nodes transmit one packet orbit at a time. The butterfly network topology is shown in Fig. 1.

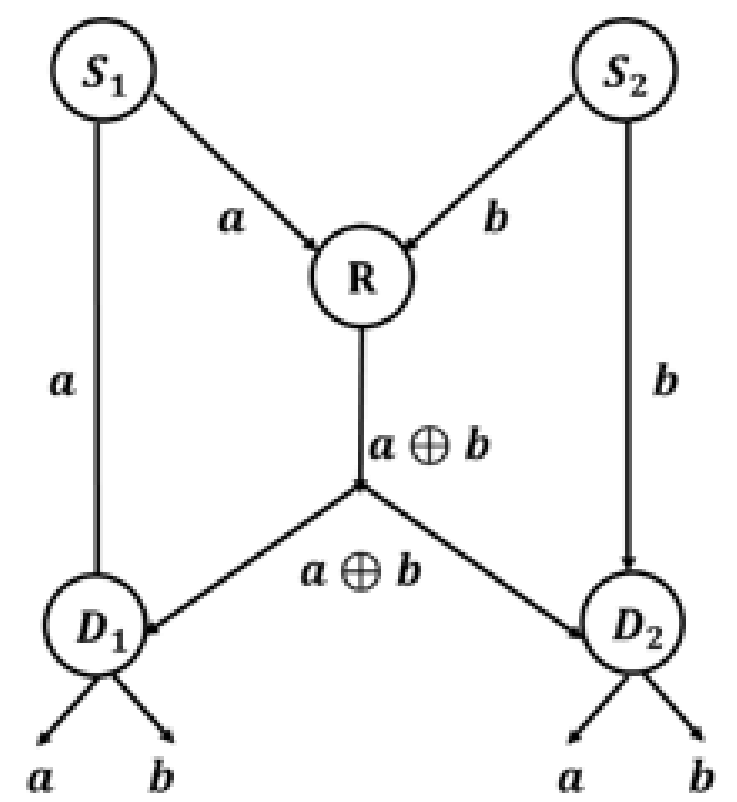

Figure 1: Butterfly network topology

The purpose here is to transfer all source packets to its destination nodes with minimum number of transmissions to boost the overall throughput of the network. The source nodes perform packet generation and transmission. The generated 
packets follow the packet structure shown in Table I.

\begin{tabular}{|c|c|c|c|c|}
\hline \multicolumn{5}{|c|}{$\begin{array}{c}\text { TABLE I } \\
\text { Simulated Packet Structure }\end{array}$} \\
\hline $\begin{array}{l}\text { Address of Source Node } \\
3 \text { Bytes }\end{array}$ & $\begin{array}{l}\text { Address of Destination Node } \\
3 \text { Bytes }\end{array}$ & $\begin{array}{l}\text { Reload } \\
100 \text { Bytes }\end{array}$ & $\begin{array}{l}\text { CRC } \\
2 \text { Bytes }\end{array}$ & $\begin{array}{l}\text { The Use of Network Coding } \\
2 \text { Bytes }\end{array}$ \\
\hline
\end{tabular}

The packet structure consists of the following:

- The first three bytes are dedicated to the source node address.

- The second three bytes are used for the destination node address.

- The payload is assumed to be 100 data bytes.

- Two bytes are used for Cyclic Redundancy Check (CRC) bits. The 16 bits CRC is generated for each packet at the source node. If the checksum at the destination node is not the same as that of the source node, the received packet is considered as an erroneous packet [16].

- The last two bytes are used as a flag for the presence of $\mathrm{NC}$ or not. Here, the binary sequence of "1's" is used when network coding is applied otherwise, a binary sequence of "0's" is used.

Let $\mathrm{a}$ and $\mathrm{b}$ be the transmitted packets from the source nodes S1 and S2, respectively. The relay node broadcasts the coded packet $r$ to all destination nodes where:

$$
a \bigoplus b
$$

Where $\bigoplus$ denote modulo- 2 or XOR operation of the corresponding bits of the packets a and $\mathrm{b}$. The destination nodes D1 and D2 receive the uncoded packets from direct transmission links (S1-D1 and S2-D2), they also store the packets considered in decoding of future packets. The destination nodes make a reply to the relay node to declare that it received the specified packet. After the reception of the coded packet at the destination nodes, the decoding process is performed as given by Eq. 2 and 3. at destination node D1

$$
a \bigoplus r=(a \bigoplus a) \bigoplus b=0 \bigoplus b=b
$$

at destination node D2

$$
r \bigoplus b=a \bigoplus(b \bigoplus b)=a \bigoplus 0=a
$$

The network coding (PLNC or NLNC (is performed in the relay node. The corresponding decoding is performed at the destination nodes D1 and D2. In PLNC, the QPSK signal received from the source nodes at the relay node are detected first to produce the corresponding detected baseband complex-valued samples $S_{1}$ and $S_{2}$ in Table II. These values are combined (summed) together to produce the PLNC coded signal. Table II shows the possible resulting signals combined by the addition of the two QPSK detected values together with the possible resulting signal $S_{T}$. NLNC is implemented at the network layer. The network layer handles data packets rather than bits. Each source node transmits its packet to both the relay node and its intended destination node. Each transmission involved the creation of the packet content first and then the modulator deals with the bits content of the packet as binary elements and proceeds as given before to produce the corresponding mapped QPSK symbol. The bit contents of the two packets, received by the relay node, are summed 
together using module-2 addition of their constituent's binary bits to produced one coded packet. The corresponding decoding is performed at the destination nodes D1 and D2.Fig. 2 shows the system model used in the work. The system may operate with an uncoded signal or with a network coded one. The output stream of the network coding/modulator block in Fig.2 is passed through the channel followed by adding an Additive White Gaussian Noise (AWGN) sample to the signal at the output of the channel. The channel may be an AWGN channel or else fading channel that models the wireless channel. The mmWave channel model considered here is a multipath fading channel with three paths representing an indoor environment.The delays and path gains of the channel are given by $(0,0.035$, and $0.612 \mu \mathrm{s})$ and $(-0.2,-18$ and -21$)$ [17] $\mathrm{dB}$, respectively, with a bandwidth of $2.5 \mathrm{MHz}$ [18] and sampling frequency of $5 \mathrm{M}$ Samples/s. The resulting Inter Symbol Interference (ISI) of the channel is dealt with by an equalizer at the receiver. The equalizer task is to remove ISI produced by the multipath channel. The received signal is then applied to the detector (demodulator) /decoder to perform detection of the signal followed by decoding.

TABLE II

Possible Values of Combined QPSK signals After PLNC

\begin{tabular}{|c|c|c|}
\hline$S_{1}$ & $S_{2}$ & $S_{T}=S_{1}+S_{2}$ \\
\hline$-1,-1$ & $-1,-1$ & $-2,-2$ \\
\hline$-1,-1$ & $-1,1$ & $-2,0$ \\
\hline$-1,-1$ & $1,-1$ & $0,-2$ \\
\hline$-1,-1$ & 1,1 & 0,0 \\
\hline$-1,1$ & $-1,-1$ & $-2,0$ \\
\hline$-1,1$ & $-1,1$ & $-2,2$ \\
\hline$-1,1$ & $1,-1$ & 0,0 \\
\hline$-1,1$ & 1,1 & 0,2 \\
\hline $1,-1$ & $-1,-1$ & $0,-2$ \\
\hline $1,-1$ & $-1,1$ & 0,0 \\
\hline $1,-1$ & $1,-1$ & $2,-2$ \\
\hline $1,-1$ & 1,1 & 2,0 \\
\hline 1,1 & $-1,-1$ & 0,0 \\
\hline 1,1 & $-1,1$ & 0,2 \\
\hline 1,1 & $1,-1$ & 2,0 \\
\hline 1,1 & 1,1 & 2,2 \\
\hline
\end{tabular}

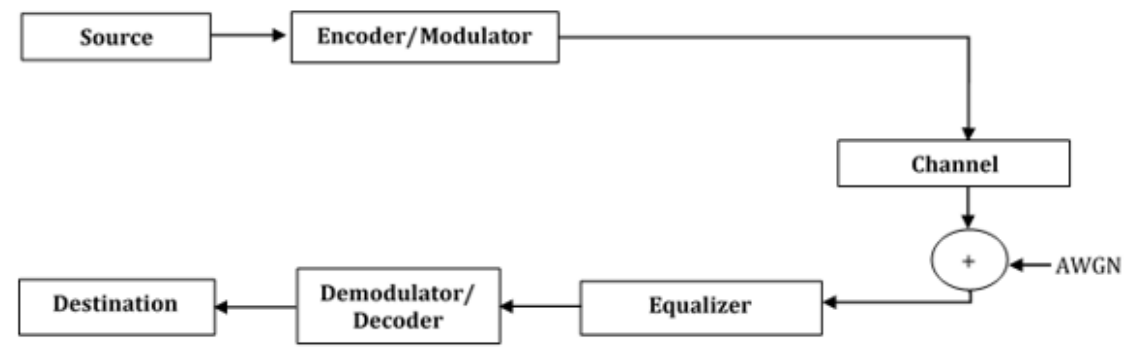

Figure 2: General system model

\section{Simulations Tests ANd Results}

Performance evaluation is performed by the simulation of the system with and without network coding. The performance measures used are Bit Error Rate (BER), Packet Error Rate (PER), and throughput versus SNR defined by Eb/No in dB. The latter is given by the ratio of the average energy per data bit (Eb) to AWGN two-sided power spectral density (No). 
The simulation results involved both non-network coded (uncoded signal) and network coded (NLNC or PLNC) signal. In either case, the modulator used is the QPSK modulator. In each test, whenever possible, SNR is varied to obtain a BER range as low as $10^{-4}$. For each SNR value, a total of at least 106 bits are transmitted from the source nodes. The corresponding detected/decoded bits at the destination nodes are then compared to those transmitted by the source nodes to determine the bit error rate. Figs. 3 and 4 present BER and PER performance for uncoded signals and the two network coding schemes (PLNC and NLNC) over different channels, respectively. Figs. 5 and 6 show the corresponding throughput performance. Fig. 3-A showed almost identical BER performances of the uncoded and NLNC systems, while PLNC relatively demonstrated clear degraded performance by about $2.5 \mathrm{~dB}$ at very low SNR. As shown in Fig. 3-B, the PLNC shows about $1.5 \mathrm{~dB}$ degradation in BER relative to uncoded signal over mmWave fading channel. The PER performances shown in Fig. 4 showed similar behavior to BER performance in Fig. 3. Both Figs. 3 and 4 showed that BER and PER performances of network coded scheme NLNC are better than those of PLNC over AWGN and mmWave fading channels. This is due to the accumulated noise that occurred in the case of PLNC. The main advantage of network coding schemes is the improved throughput. The results of the throughput performance are shown in terms of Bits/s and Packets/s. The simulated network coded schemes showed better throughput than that of the uncoded system. Figs. 5 and 6 show that the throughput of both network coded schemes (NLNC and PLNC) is better than that of an uncoded system using both Bits/s and Packets/s. The improvement of NLNC in terms of throughput performance (using Bits/s measure) is about $31 \%$ over the uncoded system for the AWGN channel as shown in Fig. 5-A. The use of network coding presents better throughput of NLNC in terms of Bits/s by about $14 \%$ over the uncoded system for mmWave channel as shown in Fig. 5-B. The improvement of NLNC in throughput using Packets/s measure over the uncoded system is about $14 \%$ as shown in Fig. 6-A for the AWGN channel. In Fig. 6-B, the throughput performance is affected by the presence of the mmWave channel, so both the network coded and uncoded systems have approximately similar throughput performance at very low SNR. At moderate and high SNR, the coded systems start to show better throughput. As for the AWGN channel, Figs. 5-B and 6-B for the mmWave channel show that the throughput in terms of Bits/s and Packets/s, respectively, in the case of NLNC and PLNC start to merge at high SNR. At high SNR, the throughput in terms of bits/s or Packets/s approaching a fixed gain of about $25 \%$ for both PLNC and NLNC.

https://ijict.edu.iq 


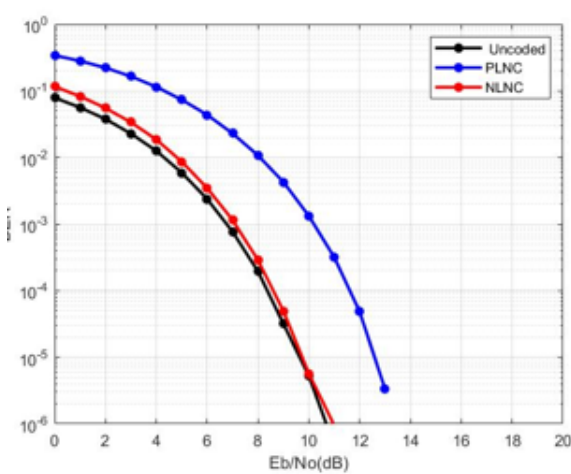

A. AWGN channel

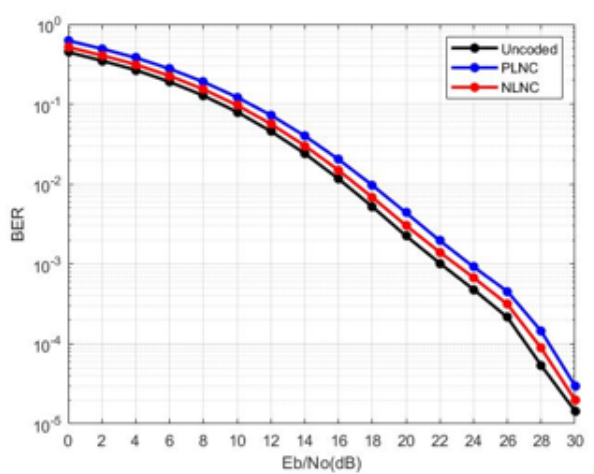

B. mm Wave channel

Figure 3: BER performance of the systems over different channels

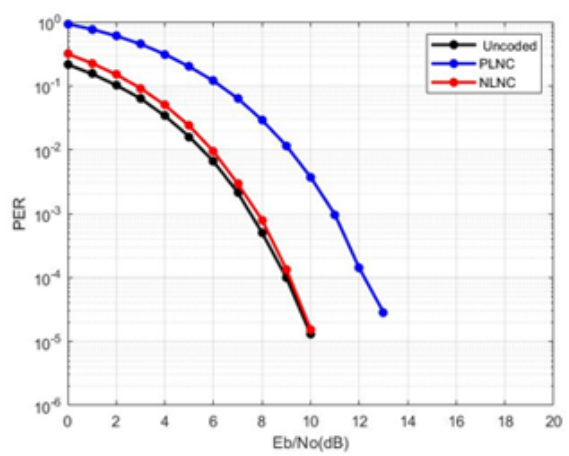

A. AWGN channel

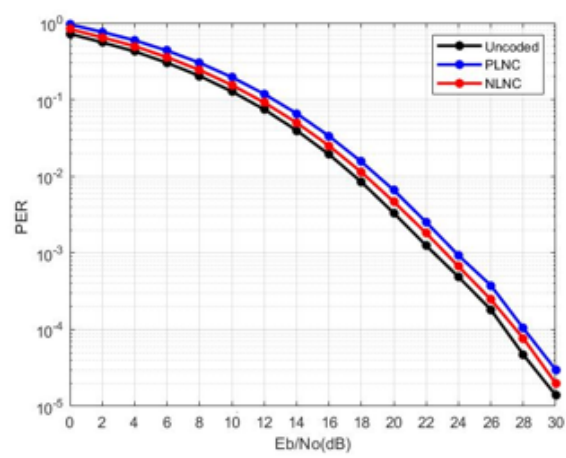

B. mmWave channel

Figure 4: PER performance of the systems over different channels

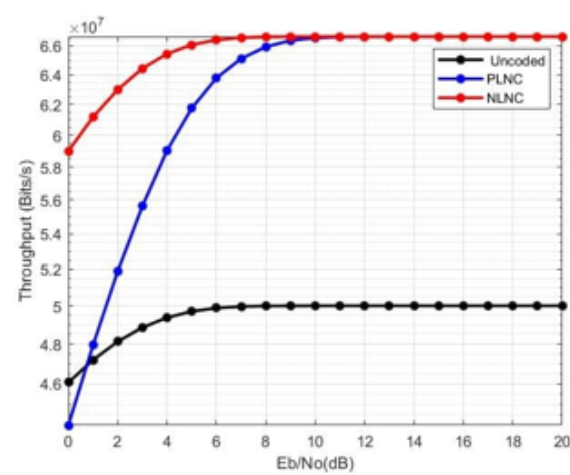

A. AWGN channel

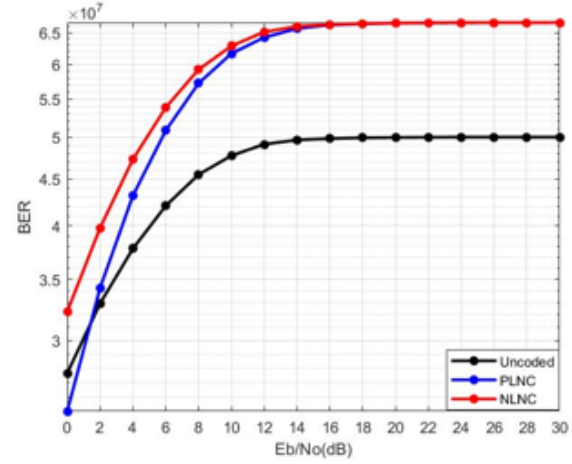

B. mm Wave channel

Figure 5: Throughput in bits/s of the systems over different channels 


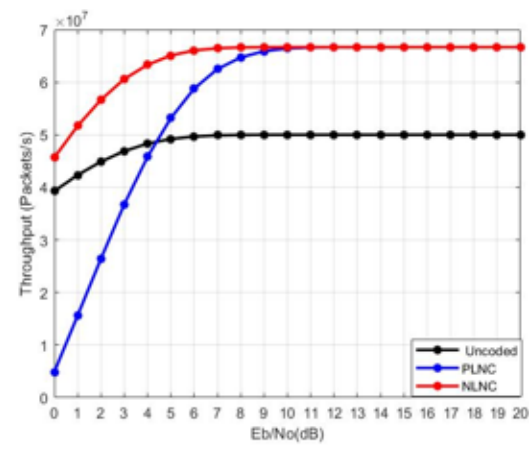

A. AWGN channel

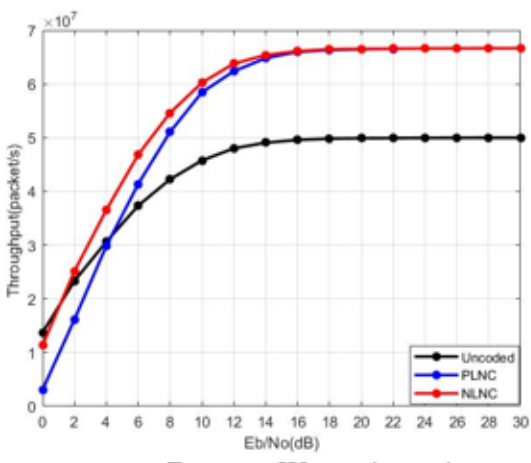

B. mm Wave channel

Figure 6: Throughput in packets/s of the systems over different channels

As shown in the previous figures, the BER and PER performance results over the AWGN channel showed that, NLNC performs better than PLNC. Unlike PLNC, the BER performance of NLNC is close to the uncoded system. This is at least due to the accumulated noise that occurred in PLNC. Further, at the relay node, the coding in both PLNC and NLNC add a sort of signal dependency among the constituent QPSK symbols and data packets, respectively. Such dependency increased the errors at the destination nodes.

\section{Conclusion}

Two network coding schemes are considered in this work. These are Physical Layer Network Coding (PLNC) and Network Layer Network Coding (NLNC). They are applied in the butterfly network topology that simulates an indoor environment using both AWGN and mmWave channel models aiming to improve the system throughput while maintaining an acceptable Bit Error Rate (BER) level. The results showed that a gain of about $25 \%$ in throughput over the uncoded system is obtained when both NC schemes PLNC and NLNC were used at high SNR. NLNC system showed better BER performance than PLNC at moderate SNR. The results also revealed that NLNC outperformed PLNC in terms of BER performance and throughput at high SNR. The main finding of the work is that the two network coding schemes can provide an improved throughput over the uncoded system at the cost of slight BER degradation. It is worthwhile to investigate the use of the considered network coding schemes in other network topologies and environments related to next-generation networks to assess the usefulness of their implementation. 


\section{REFERENCES}

[1] M. Xiao et al. , "Millimeter Wave Communications for Future Mobile Networks" , IEEE JSAC, vol. 35, no. 9, Sept. 2017.

[2] J. G. Andrews, S. Buzzi, W. Choi et al. , "What Will 5G Be ?" , IEEE Journal on Selected Areas in Communications, Vol. 32, No. 6, pp. 1065-1082, 2014.

[3] I. Gaspar and G. Wunder, "5G Cellular Communications Scenarios and System Requirements" , Tech. Rep. , 2013, https:// www.5gnow.eu/ .

[4] C. X. Wang, F. Haider, X. Gao et al., "Cellular Architecture and Key Technologies for 5G Wireless Communication Networks", IEEE Communications Magazine, Vol. 52, No. 2, pp. 122-130, 2014.

[5] X. Ge, S. Tu, G. Mao, C. X. Wang, and T. Han, "5G Ultra-Dense Cellular Networks" , IEEE Wireless Communications Magazine, vol. 23, No. 1, pp. 72-79, 2016.

[6] R. Ahlswede, N. Cai, S. Li, and R. Yrung, "Network Information Flow", IEEE Transactions on Information Theory, Vol. 46, No.4, July 2000.

[7] S. Katti, "Network Coded Wireless Architecture" , Ph.D. Thesis, Department of Electrical Engineering and Computer Science, Massachusetts Institute of Technology, Aug. 2008.

[8] C. Fragouli, E. Soljanin, "Network Coding Fundamentals" , Foundations and Trends in Networking, Vol. 2, Issue. 1, Boston, 2007.

[9] A. Alubaidy and A. K. Kadhim, "Throughput Improvement for Wireless Networks Using MIMO Network Coding", FNCES 12 conference, November 2012, Baghdad, Iraq.

[10] L. Lu, T. Wang, S. C. Liew and S. Zhang, "Implementation of Physical-Layer Network Coding" , 2012 IEEE International Conference on Communications (ICC), Ottawa, ON, 2012, pp. 4734-4740.

[11] M. K. Abboud and A. A. Kadhim, "Joint Forward Error Correction and Network Coding for Wireless Sensor Networks" , International Journal of Innovations in Engineering and Technology (IJIET), Vol. 6, No. 2, December 2015.

[12] L. F. Vieira and M. Vieira, "Network Coding for 5G Network and D2D Communication" , Conference: the 13th ACM Symposium, 2017.

[13] S. Paris, P. Kela, D. Laselva, and Q. Zhao, "Addressing Reliability Needs of Industrial Applications in 5G NR with Network Coding" , 2020 IEEE 91st Vehicular Technology Conference (VTC2020-Spring), Antwerp, Belgium, 2020.

[14] P. Chen, Z. Xie, Y. Fang, Z. Chen, S. Mumtaz and J. J. P. C. Rodrigues, "Physical-Layer Network Coding: An Efficient Technique for Wireless Communications", in IEEE Network, Vol. 34, No. 2, pp. 270-276, March/April 2020.

[15] M. Ilguy, B. Ozbek, R. Mumtaz, S. Busari and J. Gonzalez, "Coverage Analysis of Physical Layer Network Coding in Massive MIMO Systems" , in IEEE Transactions on Vehicular Technology, 2021.

[16] S. Gorshe, "CRC-16 Polynomials Optimized for Applications Using Self-Synchronous Scramblers" , 2002 IEEE International Conference on Communications, Conference Proceedings, ICC 2002 (Cat. No.02CH37333), New York, NY, USA, 2002.

[17] J. Zyren, "Overview of the 3GPP Long Term Evolution Physical Layer" , 2007, White Paper, https://www.nxp.com/docs/en/whitepaper/3GPPEVOLUTIONWP.

[18] Technical Report, "5G Study on Channel Model for Frequencies from 0.5 to $100 \mathrm{GHz}$ (3GPP TR 38.901 Version 14.0.0 Release 14) " , ETSI TR 138901 V14.0.0, France, 2017. 